\title{
Convolutional Neural Network for Edge Detection in SAR Grayscale Images
}

\author{
Mohamed A. El-Sayed ${ }^{1}$, Hamida A. M. Sennari ${ }^{2}$ \\ ${ }^{I}$ Department of Mathematics, Faculty of Science, University of Fayoum, Egypt. Assistant Prof. of CS, Taif \\ University, KSA \\ ${ }^{2}$ Department of Mathematics, Faculty of Science, Aswan University, Egypt.
}

\begin{abstract}
Traditional differential filter-based algorithms of edge detection have the advantage of theoretical strictness, but require excessive post-processing. There is not a single edge detector that has both efficiency and reliability. Neural networks are a powerful technology for classification and edge detection of the images. This paper describes a set of concrete best practices that image analysis researchers can use to get good results with neural networks. We perform an initial exploration of the effectiveness of using Convolutional Neural Networks (CNNs) for this task. CNNs exploit spatially local correlation by enforcing a local connectivity pattern between neurons of adjacent layers. At each level, the outputs of multiple networks are fused for robust and accurate estimation. It used to realize edge detection task it takes the advantage of momentum features extraction, it can process any input image of any size with no more training required, the results are very promising when compared to both classical methods and other ANN based methods. Furthermore, we employ CNNs to estimate the scale through the accurate localization of some key points. These techniques are object-independent so that the proposed method can be applied to other types of image processing such as classification and segmentation.
\end{abstract}

Keywords: Edge detection, Convolutional Neural Networks, Max Pooling

\section{INTRODUCTION}

Computer vision aims to duplicate the effect of human vision by electronically perceiving and understanding an image. Giving computers the ability to see is not an easy task. Towards computer vision the role of edge detection is very crucial as it is the preliminary or fundamental stage in pattern recognition. Edges characterize object boundaries and are therefore useful for segmentation and identification of objects in a scene. The idea that the edge detection is the first step in vision processing has fueled a long term search for a good edge detection algorithm [1].

Edge detection is a crucial step towards the ultimate goal of computer vision, and is an intensively researched subject; an edge is defined by a discontinuity in gray level values. In other words, an edge is the boundary between an object and the background. The shape of edges in images depends on many parameters: The geometrical and optical properties of the object, the illumination conditions, and the noise level in the images. Edges include the most important information in the image, and can provide the information of the object's position [2]. Edge detection is an important link in computer vision and other image processing, used in feature detection and texture analysis.

Edge detection is frequently used in image segmentation. In that case an image is seen as a combination of segments in which image data are more or less homogeneous. Two main alternatives exist to determine these segments: First, classification of all pixels that satisfy the criterion of homogeneousness. Second, detection of all pixels on the borders between different homogeneous areas.

Edges are quick changes on the image profile. These quick changes on the image can be detected via traditional difference filters [3]. Also it can be also detected by using canny method [4] or Laplacian of Gaussian (LOG) method [5]. In these classic methods, firstly masks are moved around the image. The pixels which are the dimension of masks are processed. Then, new pixels values on the new image provide us necessary information about the edge. However, errors can be made due to the noise while mask is moved around the image [6]. The class of edge detection using entropy has been widely studied, and many of the paper, for examples [7],[8],[9].

Artificial neural network can be used as a very prevalent technology, instead of classic edge detection methods. Artificial neural network [10], is more as compared to classic method for edge detection, since it provides less operation load and has more advantageous for reducing the effect of the noise [11]. An artificial neural network is more useful, because multiple inputs and multiple outputs can be used during the stage of training [12], [13].

Many edge detection filters only detect edges in certain directions; therefore combinations of filters that detect edges in different directions are often used to obtain edge detectors that detect all edges. 
This paper is organized as follows: Section 2 presents some fundamental concepts of CNN and we describe the proposed method used. Sparse connectivity of CNN and shared weights neural network will be discus in Sections 3 and 4. Another important concept of CNNs (max-pooling) will be introduce in Section 5. Experiment discussion of this paper will be present in Section 6. Simulation results and the efficiently compared with different known methods will be discus in Section 7. A comparison between Tsallis entropy based edge detection and CNN based automated edge detection present in Section 8. At last conclusion of this paper will be drawn in Section 9.

\section{CONVOLUTIONAL NEURAL NETWORK}

Typically convolutional layers are interspersed with sub-sampling layers to reduce computation time and to gradually build up further spatial and configurable invariance. A small sub-sampling factor is desirable however in order to maintain specificity at the same time. Of course, this idea is not new, but the concept is both simple and powerful [14].It combines three architectural ideas to ensure some degree of shift, scale and distortion invariance: local receptive fields, shared weights (or weights replications), and spatial or temporal sub-sampling [15]. The forward Neural Network model can be extended using constraints of specific applications. One extension is the $\mathrm{CNN}$ - that uses only local connections and shared weights. Translation invariance is a property that results from those constraints, what is very useful on image and signal processing. For instance the CNN can be used for postal code recognition [16] and phoneme recognition [17].

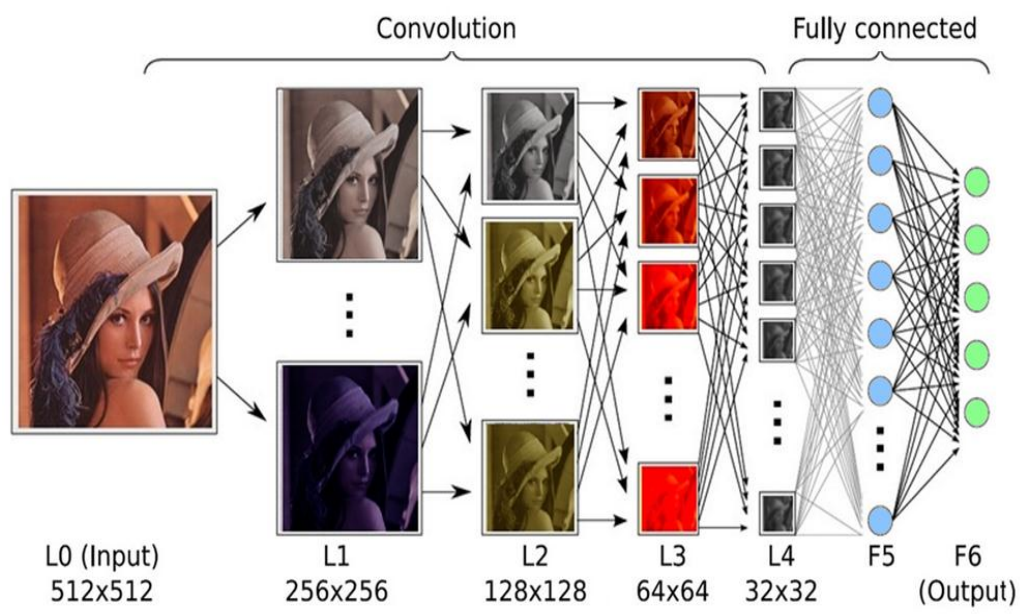

Fig. 1 CNNs structure.

The input plane receive images, each unit in a layer receives input from a set of units located in a small neighborhood in the previous layer. With local receptive fields, neurons can extract elementary visual features such as oriented edges, end points, corners (or other features such as speech spectrograms).These features are then combined by the subsequent layers in order to detect higher-order features.

CNNs are variants of MLPs which are inspired from biology. From Hubel and Wiesel's early work on the cat's visual cortex [18], we know there exists a complex arrangement of cells within the visual cortex. These cells are sensitive to small sub-regions of the input space, called a receptive field, and are tiled in such a way as to cover the entire visual field. These filters are local in input space and are thus better suited to exploit the strong spatially local correlation present in natural images. Additionally, two basic cell types have been identified: simple cells (S) and complex cells (C). Simple cells (S) respond maximally to specific edge-like stimulus patterns within their receptive field. Complex cells (C) have larger receptive fields and are locally invariant to the exact position of the stimulus.

\section{SPARSE CONNECTIVITY}

CNNs exploit spatially local correlation by enforcing a local connectivity pattern between neurons of adjacent layers. The input hidden units in the mth layer are connected to a local subset of units in the $(m-1)$ th layer, which have spatially contiguous receptive fields. We can illustrate this graphically as follows: 


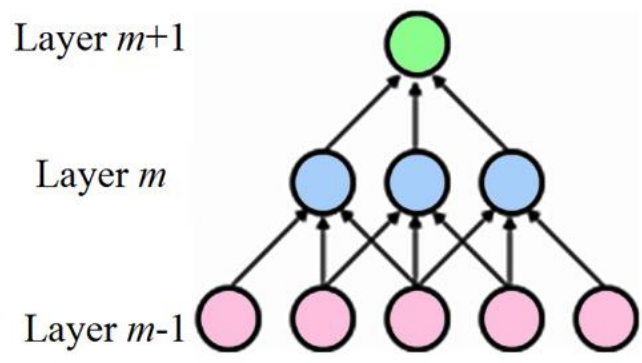

Fig. 2 Sparse Connectivity

Imagine that layer $m-1$ is the input retina. In the above, units in layer $m$ have receptive fields of width 3 with respect to the input retina and are thus only connected to 3 adjacent neurons in the layer below (the retina). Units in layer $m$ have a similar connectivity with the layer below. We say that their receptive field with respect to the layer below is also 3, but their receptive field with respect to the input is larger (it is 5). The architecture thus confines the learnt "filters" (corresponding to the input producing the strongest response) to be a spatially local pattern (since each unit is unresponsive to variations outside of its receptive field with respect to the retina). As shown above, stacking many such layers leads to "filters" (not anymore linear) which become increasingly "global" however (i.e. spanning a larger region of pixel space). For example, the unit in hidden layer $m+1$ can encode a non-linear feature of width 5 (in terms of pixel space) [19].

\section{SHARED WEIGHTS NEURAL NETWORK}

Hidden units can have shift windows too this approach results in a hidden unit that is translation invariant. But now this layer recognizes only one translation invariant feature, what can make the output layer unable to detect some desired feature. To fix this problem, we can add multiple translation invariant hidden layers:

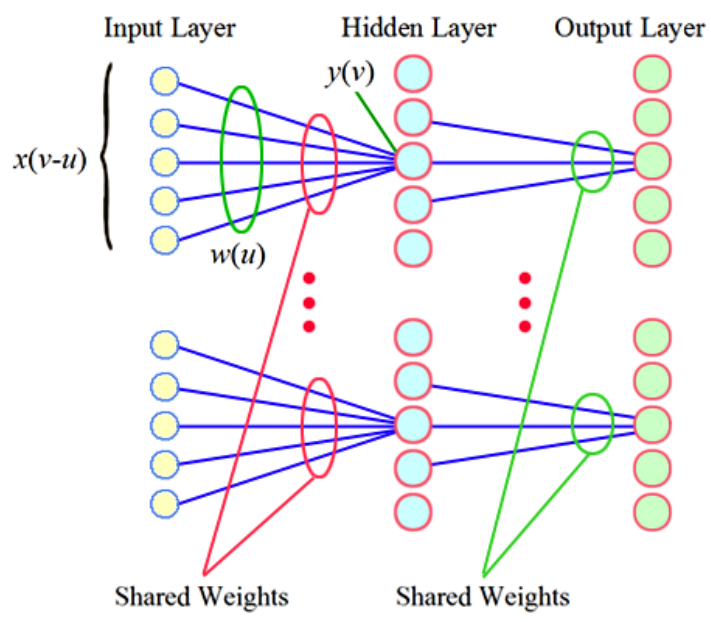

Fig. 3 Shared Weight Neural Network Edit image

A full connected Neural Network is not a good approach because the number of connections is too big, and it is hard coded to only one image size. At the learning stage, we should present the same image with shifts otherwise the edge detection would happen only in one position (what was useless).

Exploring properties of this application we assume: The edge detection should work the same way anywhere the input image is placed. This class of problem is called Translation Invariant Problem. The translation invariant property leads to the question: why to create a full connected Neural Network? There is no need to have full connections because we always work with finite images. The farther the connection, the less importance to the computation [21].

\section{MAX POOLING}

Another important concept of CNNs is that of max-pooling, which a form of non-linear down-sampling is. Max-pooling partitions the input image into a set of non-overlapping rectangles and, for each such subregion, outputs the maximum value. Max-pooling is useful in vision for two reasons:

1. It reduces the computational complexity for upper layers.

2. It provides a form of translation invariance. 
To understand the invariance argument, imagine cascading a max-pooling layer with a convolutional layer. There are 8 directions in which one can translate the input image by a single pixel. If max-pooling is done over a $2 \times 2$ region, 3 out of these 8 possible configurations will produce exactly the same output at the convolutional layer. For max-pooling over a $3 \times 3$ window, this jumps to $5 / 8$. Since it provides additional robustness to position, max-pooling is thus a "smart" way of reducing the dimensionality of intermediate representations [22].
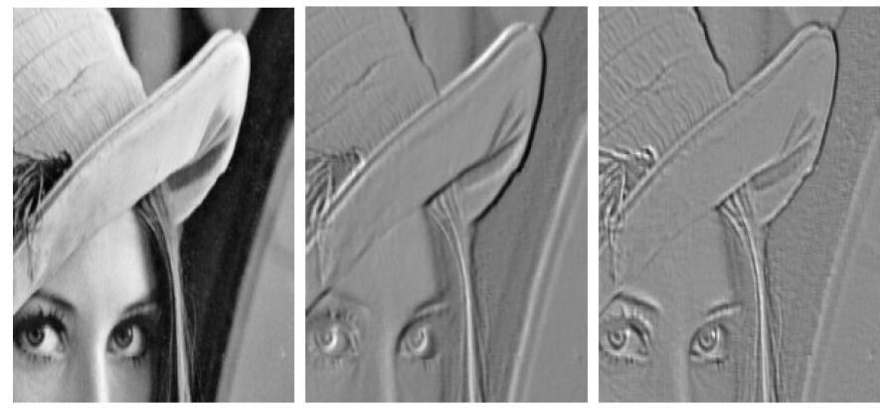

Fig. 4 Output of randomly initialized network

Sparse, convolutional layers and max-pooling are at the heart of the CNN models. While the exact details of the model will vary greatly, the Fig. below shows a graphical depiction of a model.

Implementing the network shown in Fig. 1, the input image is applied recursively to down-sampling layers reduces the computational complexity for upper layers and reduce the dimension of the input, also the network has a $3 \times 3$ receptive fields that process the sup sampled input and output the edge detected image, the randomly initialized model acts very much like an edge detector as shown in Fig. 4. The hidden layers activate for partial edge detection, somehow just like real neurons described in Eye, Brain and Vision (EBV) from David Hubel. Probably there is not "shared weights" in brains neurons, but something very near should be achieved with the presentation of patterns shifting along our field of view [23].

\section{EXPERIMENT DISCUSSION}

The training of CNNs is very similar to the training of other types of NNs, such as ordinary MLPs. A set of training examples is required, and it is preferable to have a separate validation set in order to perform cross validation and "early stopping" and to avoid overtraining. To improve generalization, small transformations, such as shift and distortion, can be manually applied to the training set. Consequently the set is augmented by examples that are artificial but still form valid representations of the respective object to recognize. In this way, the CNN learns to be invariant to these types of transformations. In terms of the training algorithm, in general, online Error Back-propagation leads to the best performance of the resulting CNN[18].

The training patterns for the Neural Network are shown in Fig. 5. Totally 17 patterns are considered, including 8 patterns for "edge" and 9 patterns for "non-edge". During training, all 17 patterns are randomly selected. For simplicity, all training patterns are binary images as shown in Fig. 5. The training process passes many stages according to training epoch's number to reach the weight values that gives the best result.
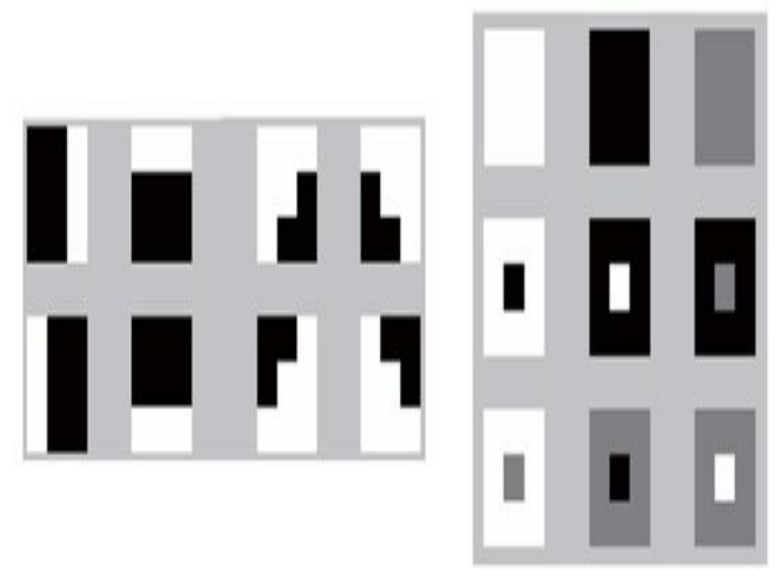

Fig. 5 Edge and non edge Training Patterns 


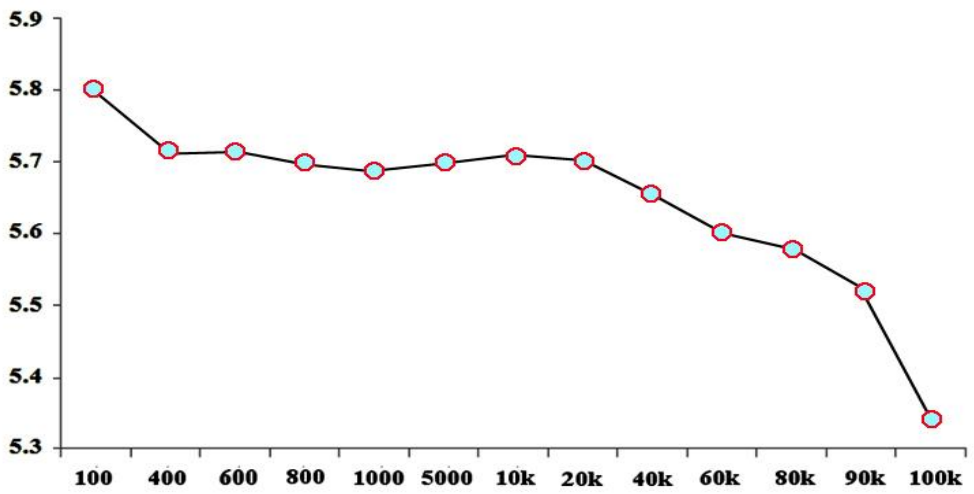

Fig. 6 PSNR changes during Training

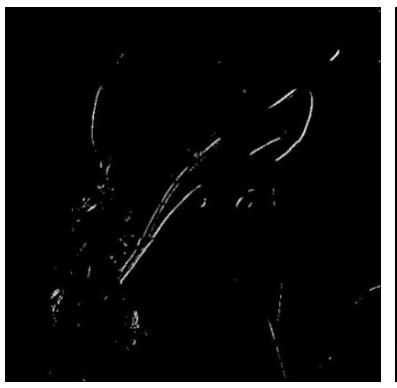

Epochs $=400$, $\mathrm{PSNR}=+5.71 \mathrm{~dB}$

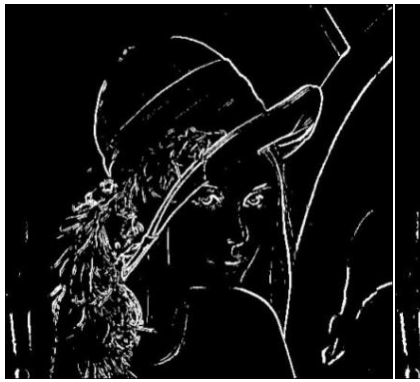

Epochs $=1000$,

$\mathrm{PSNR}=+5.70 \mathrm{~dB}$

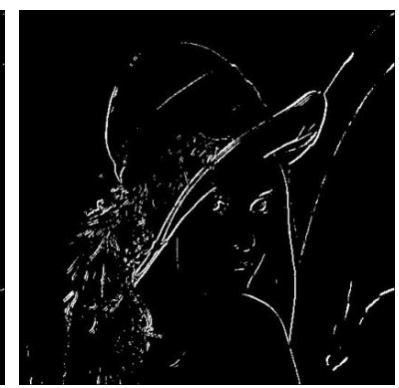

Epochs $=500$, $\mathrm{PSNR}=+5.72 \mathrm{~dB}$

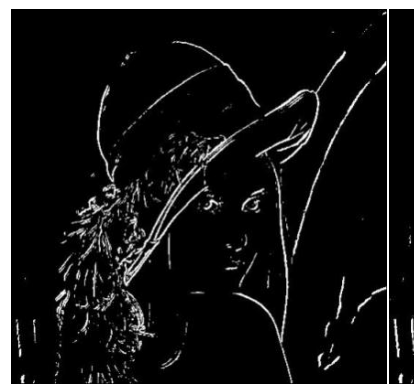

Epochs $=600$, $\mathrm{PSNR}=+5.70 \mathrm{~dB}$

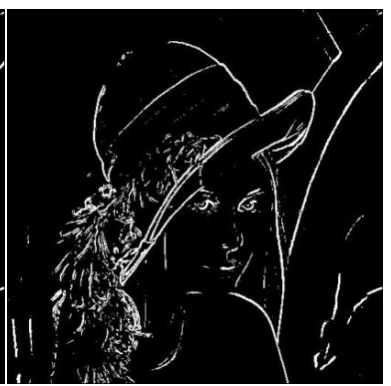

Epochs $=800$, $\mathrm{PSNR}=+5.69 \mathrm{~dB}$

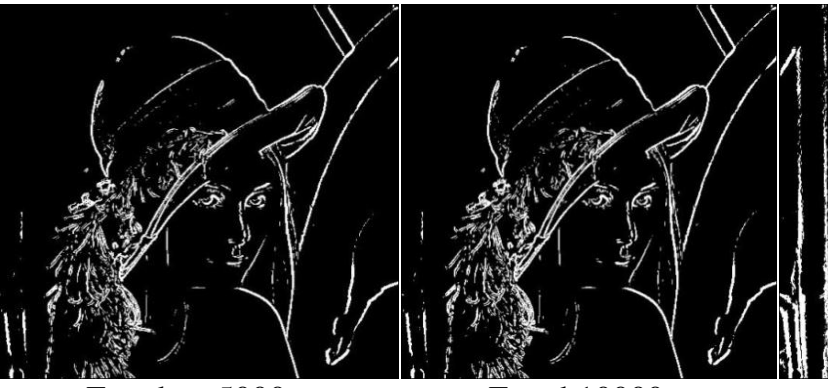

Epochs $=5000$,

PSNR $=+5.71 \mathrm{~dB}$
Epoch10000, PSNR $=+5.70 \mathrm{~dB}$

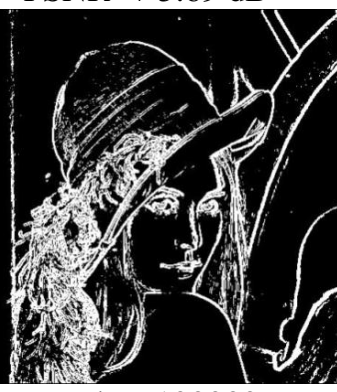

Epochs $=100000$,

$\mathrm{PSNR}=+5.33 \mathrm{~dB}$

Fig. 7 output and PSNR values for different network statues of Lena image

The epoch's number value ranges from 100 epochs to 100000 epochs as a maximum number performed. The PSNR (peak signal-to-noise ratio) is used to evaluate the network output during raising the epoch's number. The previous Fig. 6 shows the output result and its PSNR value to a test Lena image at different statuses of epoch's number value. Fig. 6 shows the changes of the edge detected output image of the proposed technique, it is obvious that the best result that gathers more expected edge pixels with least noise, PSNR = $+5.33 \mathrm{~dB}$ is reached when network was trained 100000 times, what approves the validity and efficiency of our proposed technique, the following Fig. 7 shows the changes of the noise ratio in the output edge detected Lena image when applied to the proposed system during increasing the training epochs number from 400 to 100000 epoch, a significant changes occurred when we raised the epoch number to its maximum value.

\section{SIMULATION RESULTS}

The CNN model presented in Fig. 1 is implemented using Python, VC++ and trained using sharp edge images several times to increase its ability to automatically detect edges in any test image with a variant resolution, results are compared with classical edge detectors such as (Sobel, Canny, LOG,) and technique proposed by [24] that presented a combined of entropy and pulse coupled Neural Network model for edge detection as in Fig. 9. It is obvious to notice from Fig. 9 that proposed technique achieves edge detection process efficiently compared with different known methods, where it gathers more expected edge pixels and left a little bit noise than other techniques as shown in Fig. 9. 


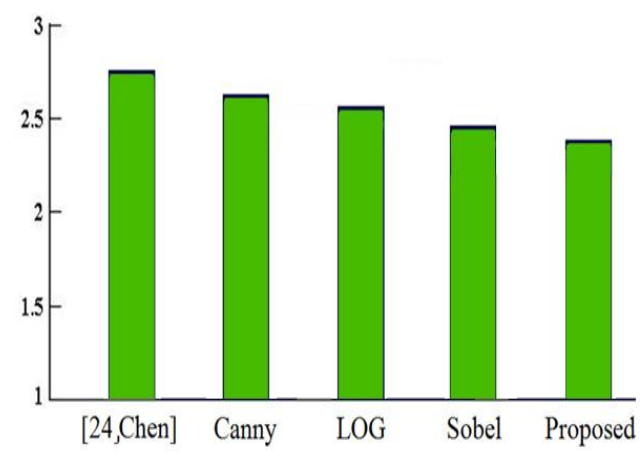

Fig. 9 SNR values compared.

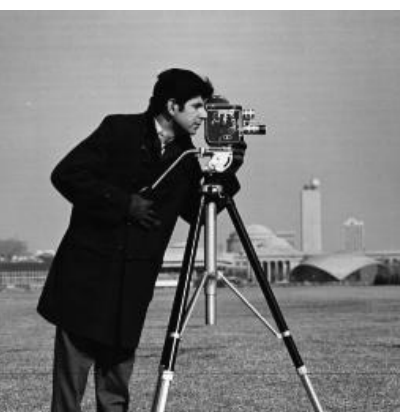

Original image

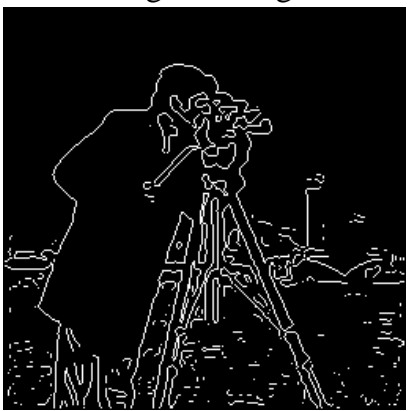

LOG, PSNR=+2.34 dB

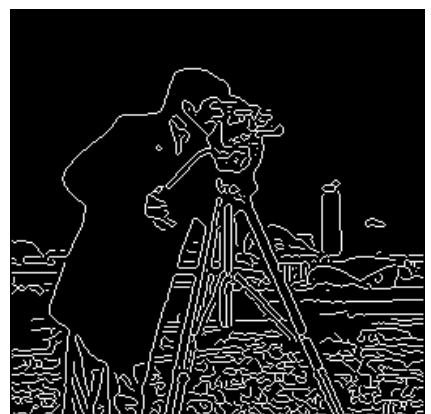

Canny, PSNR=+2.5 dB

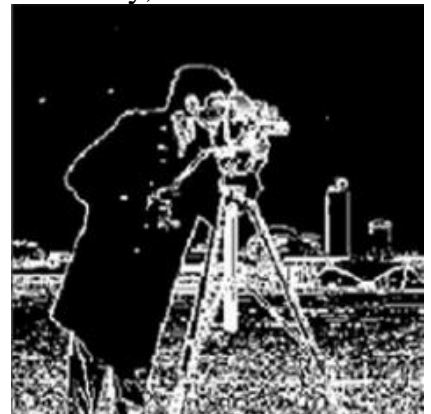

[Chen, 2010], PSNR=+2.65 dB

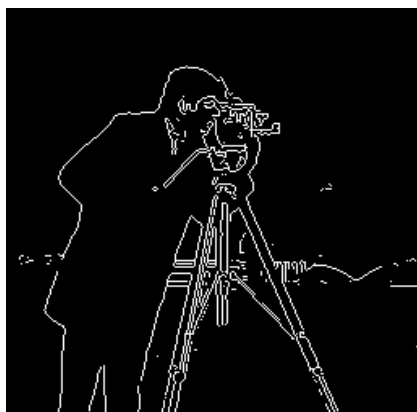

Sobel, PSNR $=+2.34 \mathrm{~dB}$

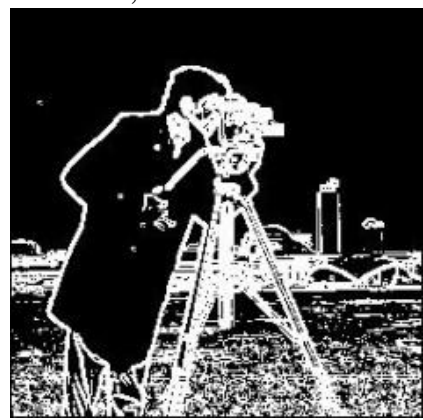

Proposed, PSNR $=+2.3 \mathrm{~dB}$

Fig. 10 comparison of different techniques Vs proposed technique of Cameraman image.

VIII. ENTROPY VS CONVOLUTIONAL NEURAL NETWORK

A comparison between Tsallis entropy based edge detection and CNN based automated edge detection is made on an image set varies between standard images and live images from different sources, with total 80 image with different sizes, intensity, lighting effects and resolution to detect the better technique that achieves the process of edge detection and the results. Fig.s (11-18) shows results of some tested image that CNN based edge detection technique results is much better than Tsallis's entropy based edge detection one. The visual scene is good to measure the edge detection technique efficiency; also PSNR can be used to detect which technique leaves more noise the following Table 1 shows the values of PSNR computed using Matlab; the original image is considered as the signal and the edge detected image.

\begin{tabular}{|c|c|c|}
\hline image & PSNR (Image, Tsallis) & PSNR (Image, CNN) \\
\hline \hline 2105 & $+5.03 \mathrm{~dB}$ & $+6.79 \mathrm{~dB}$ \\
\hline 2111 & $+5.69 \mathrm{~dB}$ & $+7.19 \mathrm{~dB}$ \\
\hline 2201 & $+4.54 \mathrm{~dB}$ & $+7.95 \mathrm{~dB}$ \\
\hline 2212 & $+1.35 \mathrm{~dB}$ & $+2.14 \mathrm{~dB}$ \\
\hline 2213 & $+11.87 \mathrm{~dB}$ & $+11.59 \mathrm{~dB}$ \\
\hline 2216 & $+4.65 \mathrm{~dB}$ & $+4.74 \mathrm{~dB}$ \\
\hline 2220 & $+1.94 \mathrm{~dB}$ & $+1.97 \mathrm{~dB}$ \\
\hline 2222 & $+0.73 \mathrm{~dB}$ & $+0.93 \mathrm{~dB}$ \\
\hline 2225 & $+2.94 \mathrm{~dB}$ & $+2.98 \mathrm{~dB}$ \\
\hline 5114 & $+7.24 \mathrm{~dB}$ & $+8.52 \mathrm{~dB}$ \\
\hline 5209 & $+0.72 \mathrm{~dB}$ & $+2.50 \mathrm{~dB}$ \\
\hline
\end{tabular}

Table 1 Some comparison between PSNR values with Tsallis entropy and CNN proposed. 
The PSNR values shows that CNN Edge detected images gathers more edges from the original image than Tsallis's ones, but Tsallis's results are about half PSNR of first ones; the following Fig. 19 shows the graphical diagram that evaluates the PSNR data in previous Table.

Fig. 11. "2105” image with $512 \times 512$ pixel.

Fig. 12. "2111" image with $512 \times 512$ pixel.

Fig. 13. "2201" image with $1024 \times 1024$ pixel.
Fig. 11. "2105" image with $512 \times 512$ pixel.

Fig. 14. "2212” image with $1024 \times 1024$ pixel.
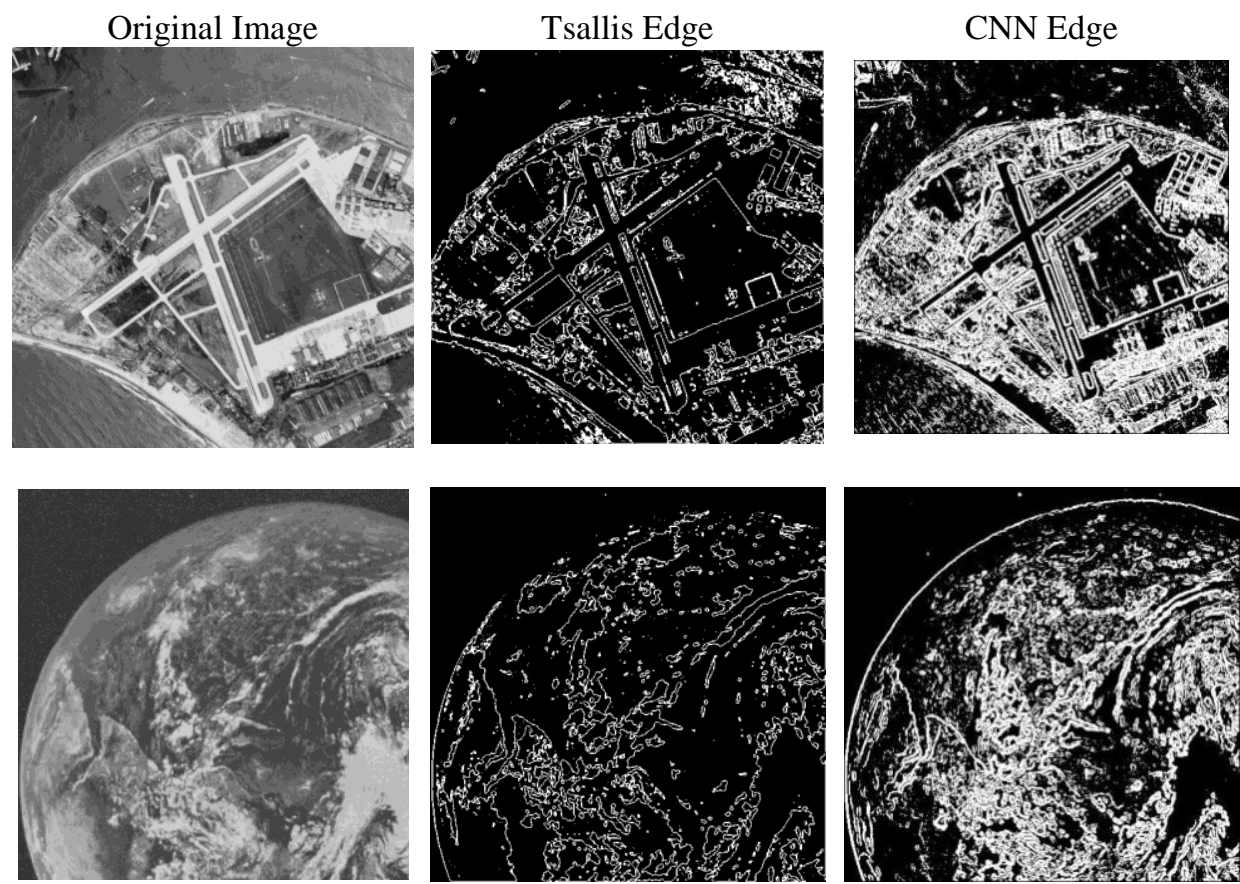

Original Image

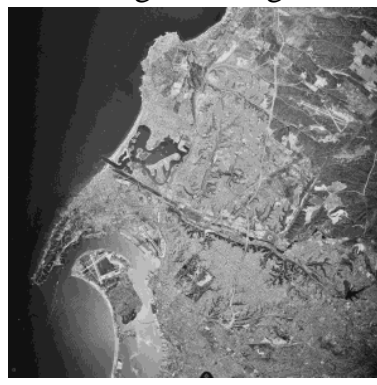

Tsallis Edge
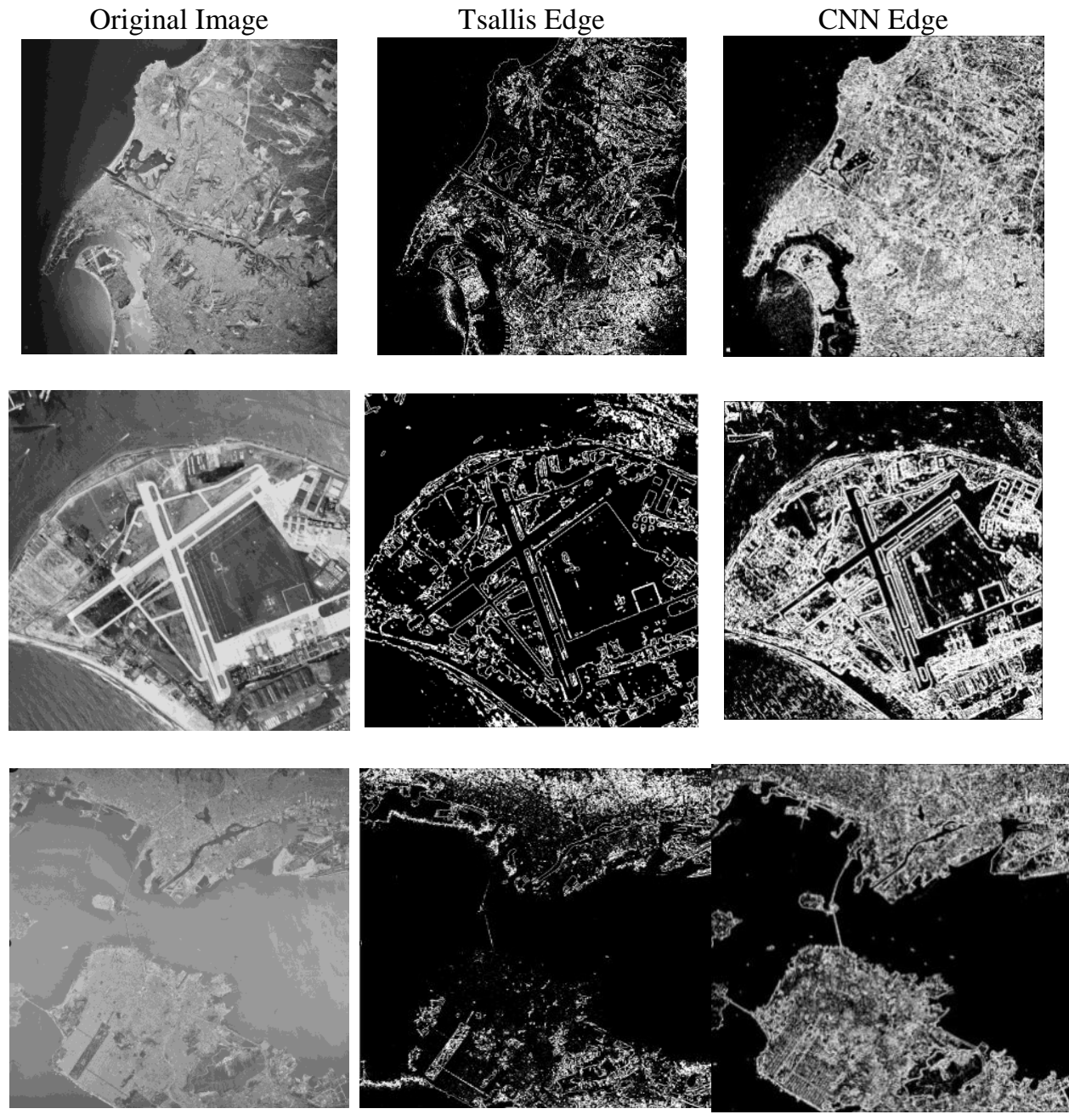

www.iosrjournals.org

$81 \mid$ Page 
Fig. 15. "2213" image with $1024 \times 1024$ pixel.
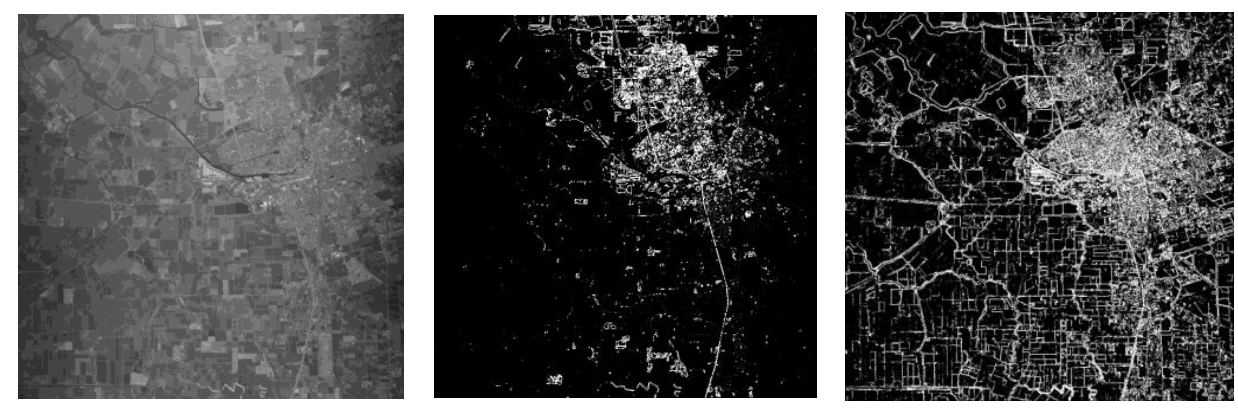

Fig. 16. "2216" image with $1024 \times 1024$ pixel.
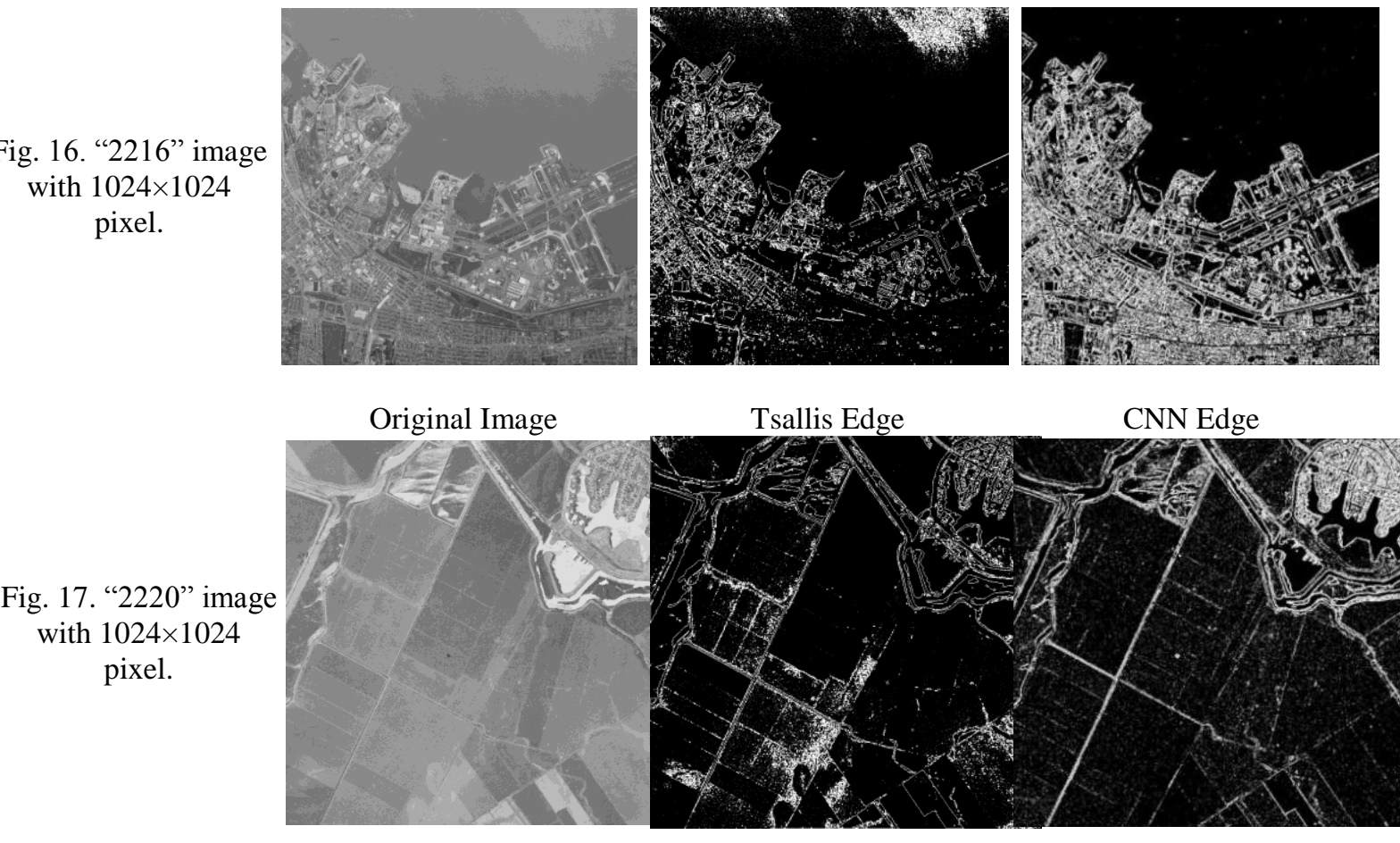

Fig. 18. "2222” image with $1024 \times 1024$ pixel.
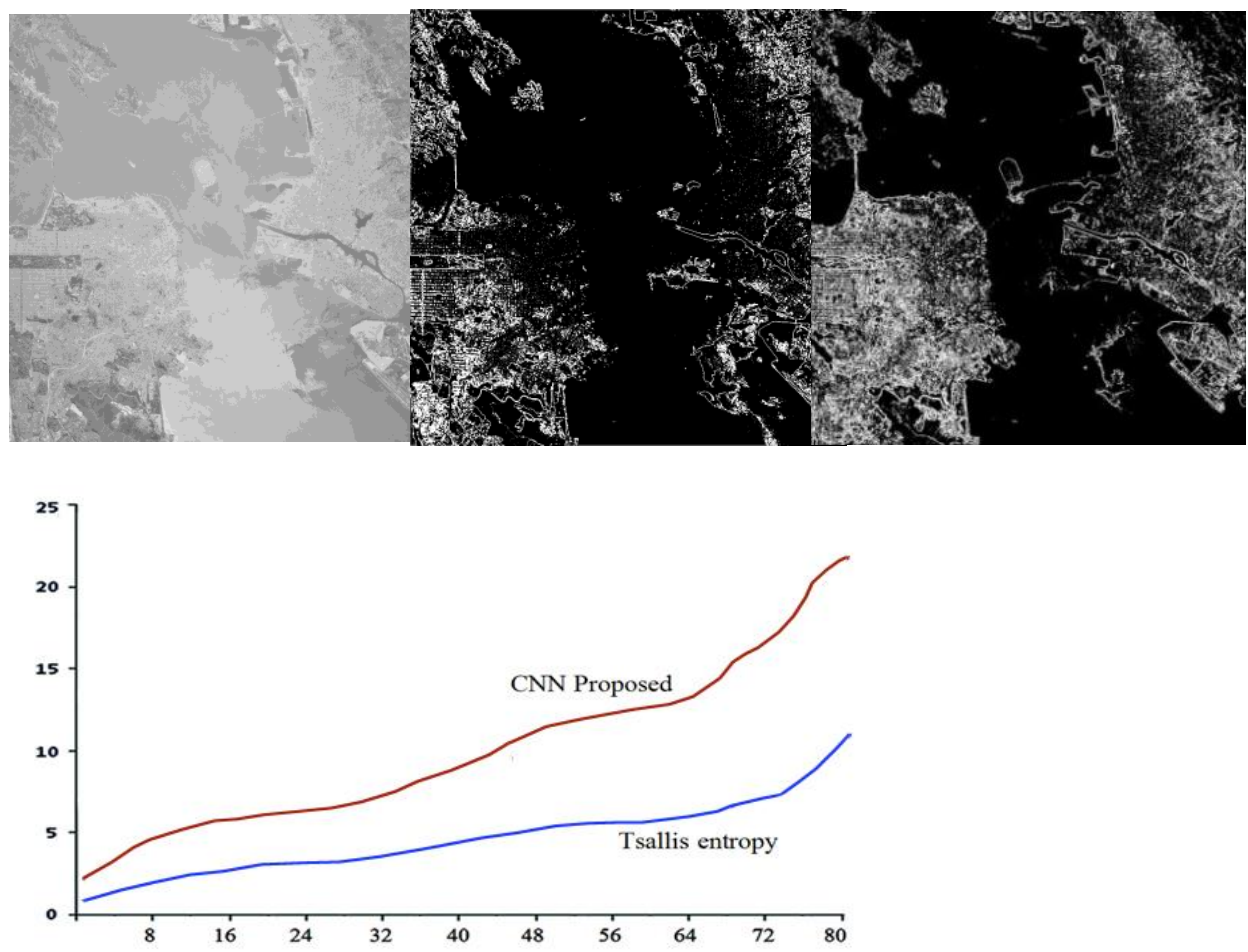

Fig. 19 PSNR values for Tsallis entropy and CNN on 80 tested images 


\section{CONCLUSION}

The CNN is used as an edge detection tool. It was trained with different edge and non edge patterns several times so that it is able to automatically detect edges in any test image efficiently. The proposed technique applied for standard images with different size, resolution, intensity, lighting effects and other conditions. The technique shows a good performance when applied on all test images. Section 8 presented a comparison between Tsallis entropy, Canny, Sobel, LOG, Chen[24] and CNN Proposed technique for edge detection to clarify which is better. the results shows that CNN better but more noisy.

\section{REFERENCES}

[1] M. Norgaard, N.K. Poulsen, O. Ravn, "Advances in derivative-free state estimation for non-linear systems," Technical Report IMM-REP-1998-15 (revised edition), Technical University of Denmark, Denmark, April 2000.

[2] L. Canny, "A computational approach to edge detection", IEEE Trans. on Pattern Analysis and Machine Intelligence, vol. 8 no. 1, pp. 679-698, 1986.

[3] D. Marr, E. Hildreth, “Theory of edge detection”, Proc. of Royal Society Landon, B(207): 187-217, 1980.

[4] R. Machuca,. "Finding edges in noisy scenes", IEEE Trans. on Pattern Analysis and Machine Intelligence, 3: 103-111, 1981.

[5] F. Rosenblatt, "The Perceptron: A Probabilistic Model for Information Storage and Organization in the Brain", Cornell Aeronautical Laboratory, Psychological Review, v65, No. 6, pp. 386-408, 1958.

[6] A. J. Pinho, L. B. Almeida, "Edge detection filters based on artificial neural networks", Pro. of ICIAP'95, IEEE Computer Society Press, p.159-164, 1995.

[7] Mohamed A. El-Sayed and Mohamed A. Khafagy, "Using Tsallis's Entropy For Edge Detection In Level Images", International Journal of Intelligent Computing and Information Science (IJICIS) Vol. 11, No.2, pp. 1-10, 2011.

[8] Mohamed A. El-Sayed, "Study of Edge Detection Based On 2D Entropy", International Journal of Computer Science Issues (IJCSI) ISSN : 1694-0814, Vol. 10, Issue 3, No 1, pp. 1-8, 2013.

[9] Mohamed A. El-Sayed , S. F.Bahgat, and S. Abdel-Khalek "Novel Approach of Edges Detection for Digital Images Based On Hybrid Types of Entropy”, International Appl. Math. Inf. Sci. 7, No. 5, pp.1809-1817, 2013.

[10] H.L. Wang, X.Q. Ye, W.K. Gu, "Training a NeuralNetwork for Moment Based Image Edge Detection" Journal of Zhejiang University SCIENCE(ISSN 1009-3095, Monthly), Vol.1, No.4, pp. 398-401 CHINA, 2000.

[11] Y. Xueli, "Neural Network and Example", Learning, Press of Railway of China, Beijing, 1996.

[12] P.K. Sahoo, S. Soltani,A.K.C. Wong, Y.C. Chen,"A Survey of the Thresholding Techniques", Computer Vision Graphics Image Process, 1988.

[13] Y. LeCun,L.Bottou,Y.Bengio, and P.Haffner."Gradient-based learning applied to document recognition”, Proceedings of the IEEE, vol. 86, pp. 2278-2324, November 1998.

[14] Yasar Becerikli, H.E. Demiray, M. Ayhan, and K. Aktas; "Alternative Neural Network Based Edge Detection",Neural Information Processing - Letters and Reviews Vol. 10, Nos. 8-9, Aug.-Sept. 2006.

[15] Hurieh Khalajzadeh, Mohammad Mansouri, Mohammad Teshnehlab, "Persian Signature Verification using Convolutional Neural Networks"; International Journal of Engineering Research and Technology (IJERT) ISSN: 2278-0181 Vol.1 Issue 2, April - 2012.

[16] LeCun, Y.; Boser, B.; Denker, J. S. et al.; "Handwritten Digit Recognition with a Back-Propagation Network". In David Touretzky. Advances in Neural Information Processing Systems 2 (NIPS *89). Denver : Morgan Kaufman, 1990.

[17] Waibel, Alexander, Hanazawa, Toshiyuki; Hinton, Geoffrey et al.; "Phoneme Recognition Using Time-Delay Neural Networks". IEEE Transactions on Acoustics, Speech and Signal Processing, v. ASSP-37, n. 3 (March), p. 328-339, 1989.

[18] Stefan Duffner "Face Image Analysis With Convolutional Neural Networks", 2007.

[19] Honglak Lee; "Tutorial on Deep Learning and Applications", NIPS Workshop on Deep Learning and Unsupervised Feature Learni, 2010.

[20] Sumedha, Monika Jenna, "Morphological Shared Weight Neural Network: A method to improve fault tolerance in Face"; International Journal of Advanced Research in Computer Engineering \& Technology Volume 1, Issue 1, March 2012

[21] Mohamed A. El-Sayed, Yarub A. Estaitia, Mohamed A. Khafagy, Automated Edge Detection Using Convolutional Neural Network, International Journal of Advanced Computer Science and Applications(IJACSA), Vol. 4, No. 10, 11-17, 2013.

[22] Jawad Nagi , Frederick Ducatelle , Gianni A. Di Caro , Dan Cires an , Ueli Meier , Alessandro Giusti ,Farrukh Nagi , Jurgen Schmidhuber, Luca Maria Gambardella; "Max-Pooling Convolutional Neural Networks for Vision-based Hand Gesture Recognition" International conference on signal and image processing applications, 2011.

[23] Ranzato; "Deep learning documentation: Convolutional Neural Networks" Copyright 2008--2010, LISA lab. Last updated on Dec 03, 2012.

[24] Jiansheng Chen, Jing Ping Heand Guangda Su, "Combining image entropy with the pulse coupled Neural Network in edge detection", ICIP 2010. 